\title{
PRINCÍPIOS BÁSICOS E APLICAÇÃO DA IONTOFORESE NA PENETRAÇÃO CUTÂNEA DE FÁRMACOS
}

\author{
Taís Gratieri, Guilherme Martins Gelfuso e Renata Fonseca Vianna Lopez* \\ Faculdade de Ciências Farmacêuticas de Ribeirão Preto, Universidade de São Paulo, Av. do Café, s/n, 14040-903 Ribeirão Preto \\ - SP, Brasil
}

Recebido em 7/11/06; aceito em 20/9/07; publicado na web em 31/7/08

\begin{abstract}
BASIC PRINCIPLES AND APPLICATIONS OF IONTOPHORESIS FOR CUTANEOUS PENETRATION OF DRUGS. Physicochemical constraints severely limit the number of molecules that can be considered as candidates for transdermal delivery. Iontophoresis is a non-invasive technique in which a weak electric current is used to enhance the penetration of molecules into or through the skin. In this review the underlying mechanisms that drive iontophoresis and the impact of key experimental parameters - namely, formulation, drug concentration and $\mathrm{pH}$ - on iontophoretic delivery efficiency are discussed. In the final section some devices that are currently commercialized are also described.
\end{abstract}

Keywords: iontophoresis; topic and transdermal delivery; electrorepulsion.

\section{INTRODUÇÃO}

A aplicação da eletricidade em diferentes terapias remonta aos gregos, tendo sido explorada antes mesmo de seu descobrimento. ${ }^{1}$ Existem relatos do físico grego Aetius sobre a indicação de choques utilizando peixe elétrico para o tratamento de gota e hemorróida. Wiliam Gilbert, físico da rainha Elizabeth I, também se interessou pelo uso da eletricidade em tratamentos e publicou o livro "De Magneto", por volta de 1600. Mas foi em 1800 que Alexandro Volta provou que o fluxo de corrente elétrica ocorre entre dois metais semelhantes, quando colocados em contato, e atribuiu a este fenômeno a contração dos músculos da perna de sapo, observada em 1780 por Galvani. ${ }^{2}$

A aplicação de uma corrente elétrica fraca para transferir substâncias carregadas através de membranas biológicas, ou seja, a iontoforese, não é uma técnica nova. Ela foi primeiramente descrita por Veratti em 1748 e vem sendo modificada ao longo dos anos de acordo com as necessidades e capacidade tecnológica. ${ }^{3}$ No final do século XIX, Morton escreveu um livro sobre a cataforese de íons nos tecidos, isto é, a migração de cátions de um eletrodo positivo em direção a um eletrodo de polaridade negativa. Neste livro o autor descreve um experimento conduzido em seu próprio braço. Nele, grafite em pó foi colocada em contato com um eletrodo positivo e a aplicação de uma corrente elétrica provocou o aparecimento de pequenas manchas pretas no local, que persistiram por várias semanas. No início do século XX, Leduc mostrou que a iontoforese poderia ser usada para levar princípios ativos através da pele usando dois coelhos conectados em série a um gerador de corrente. Soluções de estricnina e de cianeto foram colocadas em contato com a pele dos animais e uma corrente elétrica foi aplicada. Sem a aplicação da corrente elétrica nada aconteceu aos coelhos. No entanto, após a aplicação da corrente, o coelho ao qual foi aplicada estricnina apresentou convulsões tetânicas, enquanto o segundo morreu apresentando sintomas de envenenamento por cianeto. ${ }^{2}$ Desde então a iontoforese é utilizada e extensivamente investigada para promover a liberação de fármacos através de vários epitélios, como mucosas, ${ }^{4-7}$ cérvix, ${ }^{8}$ olhos, ${ }^{9-13}$ unhas ${ }^{14,15}$ e, principalmente, pele ${ }^{16-24}$ no tratamento de diversas patologias.

A administração de fármacos na pele, seja para um tratamento

\footnotetext{
*e-mail: rvianna@fcfrp.usp.br
}

local (tópico) ou sistêmico (transdérmico), baseia-se na difusão do fármaco através das diversas camadas da epiderme. A camada mais superficial deste tecido, o estrato córneo, mantém o conteúdo de água no organismo mesmo em condições climáticas variáveis, bem como limita a absorção de substâncias tóxicas do ambiente. Sendo assim, o estrato córneo apresenta excelentes propriedades de barreira para a penetração de fármacos na pele, o que limita a liberação de um grande número de substâncias por esta via. A maioria dos fármacos liberados pelos sistemas transdérmicos convencionais são moléculas pequenas, potentes e relativamente lipofílicas. A administração cutânea de fármacos polares e que apresentam carga é particularmente difícil, devido à lipofilicidade intrínsica do estrato córneo. ${ }^{25-27} \mathrm{~A}$ iontoforese apresenta o potencial de vencer várias limitações associadas à liberação transdérmica, como promover a penetração de moléculas polares e de grande massa molecular, ${ }^{28-32}$ aumentar a liberação de substâncias de meia-vida curta diretamente nos tecidos, ${ }^{33}$ além de permitir um melhor controle no transporte de fármacos, garantindo dosagem adequada e boa aceitação pelo paciente. ${ }^{34,35} \mathrm{~A}$ baixa variabilidade biológica paciente/paciente promovida pelo controle da corrente elétrica é outra vantagem da iontoforese quando comparada à administração passiva (sem corrente elétrica) de substâncias. ${ }^{36}$ Singh et $a l .{ }^{37}$ estudaram o efeito da iontoforese de uma solução salina na função barreira da pele e possíveis ocorrências de irritação local em pessoas de quatro grupos étnicos, analisando a perda de água transepidermal (TEWL), a capacitância e temperatura cutânea. Eles não observaram diferenças significativas dos parâmetros analisados entre os diferentes grupos citados após $4 \mathrm{~h}$ de iontoforese. Estes dados sugerem, portanto, que a iontoforese de um determinado fármaco deve levar à penetração cutânea de quantidades semelhantes da substância administrada, independente do grupo étnico ao qual o paciente pertença, uma vez que os parâmetros estudados por Singh et al. influenciam diretamente a penetração cutânea de substâncias. Power $^{34}$ descreveu um sistema de liberação iontoforética de fentanil controlado pelo paciente para tratamento de dor pós-operatória. Nos estudos clínicos de fase III concluiu-se que a absorção do fentanil não foi afetada por características dos pacientes, como idade, gênero, etnia ou massa corpórea.

Apesar de desde 1900 se saber que a iontoforese aumenta a penetração de fármacos na pele, ela começou a ser estudada com maior atenção somente em meados de 1980. Existem alguns fatores principais 
que contribuíram para este interesse renovado pela técnica. O primeiro deles está relacionado com a terapia transdérmica. A aceitabilidade e o sucesso financeiro dos dispositivos transdérmicos passivos de nicotina, fentanil, nitroglicerina e estradiol estimulou o interesse das indústrias farmacêuticas em trabalhar com outros fármacos que pudessem ser liberados pela via transdérmica. ${ }^{38} \mathrm{O}$ avanço da biotecnologia, com o descobrimento de grande número de peptídeos e proteínas com importância terapêutica indiscutível, mas muito grandes para serem liberados passivamente através da pele, também contribuiu para um maior interesse no uso da iontoforese. ${ }^{39}$

Um grande avanço nas possibilidades de utilização da iontoforese foi possível após o desenvolvimento de microprocessadores, quando, em 1971, a Intel Corporation introduziu no mercado o primeiro microprocessador. $\mathrm{O}$ avanço na tecnologia e indústria de microeletrônicos tornou possível miniaturizar os componentes eletrônicos programáveis do dispositivo iontoforético a um custo mais baixo. Estudos demonstram que através de microcontroladores eletrônicos programáveis pode-se obter dispositivos com esquemas posológicos complexos. ${ }^{34,40-42}$ Dispositivos iontoforéticos bem desenvolvidos são capazes, por exemplo, de mimetizar a secreção fisiológica natural de um hormônio como se fossem glândulas endócrinas artificiais, liberando o fármaco de maneira pulsátil ou contínua enquanto o paciente dorme. O hormônio de liberação do hormônio luteinizante (LHRH), por exemplo, pode ser liberado de maneira contínua para o tratamento do câncer de próstata e de maneira pulsátil para o tratamento da infertilidade..$^{43}$ Além disso, o controlador eletrônico pode ser ligado ou desligado quando necessário e, como o fármaco só é liberado na presença da corrente elétrica, o simples desligamento do controlador eletrônico provocará a diminuição do nível plasmático do fármaco. Esta característica permite a utilização da iontoforese na administração de opióides. Desta forma, quando o paciente sente dor ele ativa o dispositivo, o qual libera imediatamente uma grande quantidade do fármaco na corrente sanguínea. ${ }^{44,45}$ Atualmente, um dispositivo controlado pelo paciente para liberação iontoforética de fentanil foi aprovado nos Estados Unidos e Europa para tratamento de dor pós-operatória aguda e moderada em adultos hospitalizados. Vários estudos clínicos comprovam a equivalência terapêutica entre o uso deste dispositivo e a administração intravenosa de morfina. . $^{34,35,46,47}$ Essa grande versatilidade e facilidade de aplicação fazem com que o paciente siga corretamente a terapia que lhe foi prescrita, com baixo índice de desistência.

Para se conseguir uma permeação adequada de um fármaco por iontoforese, ou seja, para que o fármaco atinja a camada desejada da membrana ou atravesse-a e caia na circulação com concentração plasmática e velocidade de liberação controladas, aspectos químicos, físicos e fisico-químicos da formulação que será submetida à iontoforese devem ser levados em consideração. Sendo assim, o objetivo deste artigo é possibilitar o entendimento de princípios básicos da iontoforese, revisar o que se conhece dos principais mecanismos envolvidos e descrever como alguns parâmetros experimentais, tais como a concentração de fármaco e o pH da formulação, podem influenciar na liberação iontoforética de uma substância. Além disso, serão apresentadas informações sobre dispositivos iontoforéticos inovadores aprovados recentemente pelo FDA. O entendimento de princípios básicos da iontoforese é fundamental para a otimização da eficiência da corrente elétrica e, conseqüentemente, para o desenvolvimento de um sistema de liberação adequado.

\section{PRINCÍPIOS BÁSICOS}

A iontoforese é uma técnica não-invasiva, baseada na aplicação de uma corrente elétrica de baixa intensidade para facilitar a liberação de uma variedade de fármacos, carregados ou não, através de membranas biológicas, rumo à corrente sangüínea. ${ }^{31}$

A corrente elétrica é fornecida por uma fonte de energia ou bateria e distribuída com o auxílio de um eletrodo positivo (ânodo) e um eletrodo negativo (cátodo) através de uma solução eletrolítica que segue para a pele e vai para o sistema circulatório. ${ }^{22-24}$ Quando a corrente é aplicada, os cátions presentes na solução em contato com o ânodo se movem em direção ao cátodo, enquanto os ânions presentes no cátodo se movem na direção oposta. A presença de cada tipo de íon (cátions e ânions) ou moléculas carregadas no sistema conduz uma fração de corrente elétrica denominada número de transporte. A razão máxima do transporte de um dado íon ocorre quando o número de transporte para o íon é igual a 1, isto é, quando o íon sozinho é capaz de conduzir $100 \%$ da corrente através da membrana. ${ }^{48-50}$ Para otimizar o transporte iontoforético desses íons é necessário adequar dispositivos iontoforéticos e formulações compatíveis com eles, isto porque o transporte de íons é dependente de vários fatores relacionados ao dispositivo iontoforético e à formulação utilizada. Sendo assim, o fluxo iontoforético de um fármaco pode ser aumentado através da alteração de fatores elétricos, como a densidade e a natureza da corrente e o tipo de eletrodo utilizado. Na formulação podem ser modificados a concentração do fármaco, a concentração de outros íons, o pH e a força iônica. ${ }^{26,51-54}$

Os eletrodos utilizados nos experimentos de iontoforese devem ser escolhidos para garantir a eletroneutralidade do sistema e os processos eletroquímicos desejados na interface eletrodo-solução e, assim, maximizar a liberação iontoforética do fármaco em estudo. Desta maneira, os eletrodos devem ter propriedades bem delineadas, pois, de seu comportamento depende a condução estável da corrente elétrica necessária para a liberação controlada do fármaco por iontoforese. Eles devem ser capazes de sustentar uma alta densidade de corrente sem causar alterações no $\mathrm{pH}$ da solução que está em contato, perda de material da superfície do eletrodo ou formação de bolhas de gás. Devem ser também mecanica e quimicamente estáveis na solução que estão em contato e relativamente fáceis de serem obtidos. E por fim, a substância submetida à corrente elétrica com tais eletrodos não deve ser alterada pelas propriedades químicas desses eletrodos. ${ }^{55}$

Os eletrodos convencionalmente empregados na iontoforese podem ser classificados como inertes (metais como aço inoxidável, platina ou alumínio) ou reversíveis $(\mathrm{Ag}$ e $\mathrm{AgCl})$, sendo os últimos mais estudados e utilizados. Os eletrodos inertes provocam a hidrólise da água, produzindo íons hidroxila e hidrônio, que podem competir com o fármaco ionizado, reduzindo o número de transporte deste e, dessa forma, sua penetração pela pele. Além disso, a eletrólise da água pode alterar o $\mathrm{pH}$ da solução doadora, degradar o fármaco ou alterar sua ionização, prejudicando assim o controle de sua liberação. A diminuição do $\mathrm{pH}$ pode também causar uma sensação de ardência no local de aplicação do fármaco.

Já os eletrodos reversíveis de $\mathrm{Ag} / \mathrm{AgCl}$ são mais funcionais do que os eletrodos inertes devido a sua cinética extremamente rápida e à relativamente alta condutividade do cloreto de prata. Eles não causam variação de $\mathrm{pH}$, pois suas trocas eletroquímicas ocorrem em uma voltagem inferior à necessária para que haja a eletrólise da água. ${ }^{1,56}$

A Figura 1 ilustra a aplicação de um potencial elétrico e o deslocamento da corrente elétrica pelo circuito na presença de eletrodos reversíveis de $\mathrm{Ag}$ e $\mathrm{AgCl}$.

Pode-se observar na Figura 1 que as reações eletroquímicas no ânodo (eletrodo de $\mathrm{Ag}$ ) necessitam da presença de íons $\mathrm{Cl}^{-}$, os quais reagem com a prata e formam cloreto de prata. Este, por sua baixa solubilidade, é simultaneamente depositado na superfície do eletrodo liberando um elétron (Equação 1). Para manter a neutralidade elétrica do sistema, ou um cátion deixa este compartimento e move-se através da pele, ou um ânion presente na pele migra em direção ao ânodo. 


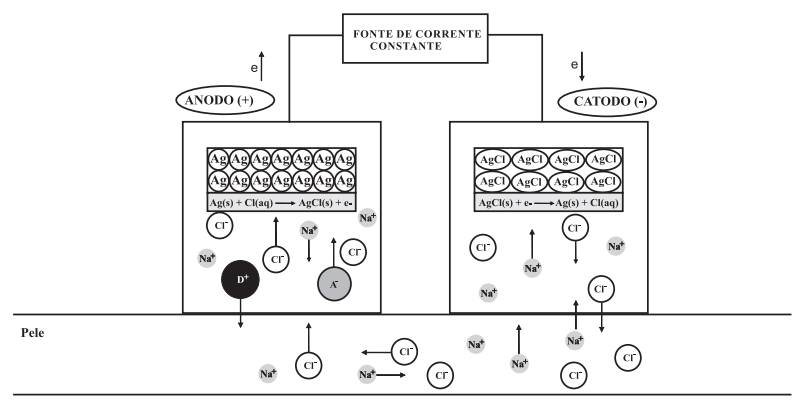

Figura 1. Iontoforese utilizando sistema de eletrodos de $\mathrm{Ag} / \mathrm{AgCl}$. O ânodo contém um fármaco ionizável $D^{+}$e seu contra íon $\mathrm{A}^{-}$e $\mathrm{Na}^{+} \mathrm{Cl}$. Adaptado da ref. 56

$\mathrm{Ag}+\mathrm{Cl}^{-} \rightarrow \mathrm{AgCl}+\mathrm{e}^{-}$(ânodo)

$\mathrm{AgCl}+\mathrm{e}^{-} \rightarrow \mathrm{Ag}^{+}+\mathrm{Cl}^{-}$(cátodo)

Já no cátodo (eletrodo de $\mathrm{AgCl}$ ), o cloreto de prata é reduzido com a chegada do elétron liberado pela oxidação da prata no ânodo e há formação de prata sólida. A prata permanece depositada no eletrodo e ocorre a liberação de íons $\mathrm{Cl}^{-}$para a solução (Equação 1). Novamente, a eletroneutralidade do sistema é mantida ou com a entrada de um cátion proveniente da pele, ou com a perda de um ânion da solução eletrolítica para a pele..$^{56,57}$

O circuito elétrico é, portanto, completado pelos íons inorgânicos presentes na pele, principalmente $\mathrm{Na}^{+} \mathrm{e} \mathrm{Cl}-\mathrm{e}$, desta forma, esses íons podem interferir na eficiência do transporte de corrente pelo fármaco. ${ }^{1}$ Se não há suplemento adequado de íons $\mathrm{Cl}^{-}$para o eletrodo de $\mathrm{Ag}$, o próprio eletrodo pode liberar íons para transportar a corrente elétrica com concomitante evolução de oxigênio, diminuindo o pH do sistema. Neste caso a conseqüente liberação de íons $\mathrm{Ag}^{+}$pode causar irritações cutâneas ${ }^{58}$ e competição com o fármaco pelo transporte de corrente elétrica.

\section{VIAS DE PERMEAÇÃO CUTÂNEA}

A pele é um dos maiores órgãos do corpo humano, pesando, em um adulto, por volta de $4 \mathrm{~kg}$, cobrindo uma superfície de cerca de $2 \mathrm{~m}^{2}$ e recebendo aproximadamente $1 / 3$ da circulação sanguínea do corpo. Com a espessura de apenas alguns milímetros $(2,97 \pm 0,28 \mathrm{~mm})$, a pele separa a rede de circulação sanguínea e os demais órgãos do corpo do ambiente externo, ajudando a manter a temperatura corporal, evitando a perda excessiva de água pelo corpo, além de proteger o indivíduo contra a entrada de agentes químicos e ambientais danosos, particularmente infecciosos (bactérias e vírus), impurezas e radiação solar. Além disso, possui funções metabólicas, imunológicas e táteis. ${ }^{59}$ Observam-se ainda várias estruturas anexas, que são os pêlos, as unhas e as glândulas sudoríparas e sebáceas. ${ }^{60}$

A pele apresenta-se constituída por uma porção epitelial de origem ectodérmica, a epiderme, e uma porção conjuntiva de origem mesodérmica, a derme. O limite entre a epiderme e a derme não é regular, apresentando saliências e reentrâncias das duas camadas que imbricam e se ajustam entre si. Abaixo e em continuidade com a derme está a hipoderme, que, embora tenha a mesma origem da derme, não faz parte da pele. ${ }^{60}$ Este tecido subcutâneo funciona como uma reserva de gordura, com propriedades isolantes e protetoras; não possui um papel na penetração cutânea de fármacos porque se situa abaixo do sistema vascular. ${ }^{37} \mathrm{O}$ acúmulo de gordura neste tecido representa um dos fatores contribuintes para a força tênsil da pele.

A derme é formada por uma rede de fibras de colágeno de uma espessura aproximadamente uniforme, que são responsáveis pelas propriedades elásticas da pele. A camada superior da derme é formada por papilas que se projetam em direção à epiderme e que contêm vasos sanguíneos e linfáticos, as terminações nervosas e os apêndices cutâneos, como folículos pilosos, glândulas sebáceas e sudoríparas. ${ }^{59}$

A epiderme pode ser dividida em duas partes: epiderme viável, parte mais interna na qual as células se proliferam, sofrem alterações e dão origem às células mortas do estrato córneo; estrato córneo, parte mais superficial, de grande importância para os estudos de penetração cutânea. ${ }^{28,61}$

O estrato córneo é formado por uma estrutura bifásica de lipídioproteína e tem aproximadamente de 10 a 20 m de espessura, ${ }^{61}$ sendo composto por células anucleadas (corneócitos) dispersas em uma matriz rica em lipídios não polares. Essa matriz é constituída principalmente por ceramidas (18\%), ácidos graxos livres (19\%), esteróides (14\%) e triacilgliceróis (25\%). O estrato córneo requer no mínimo $10 \%$ de umidade para que sua flexibilidade seja mantida, e a camada de lipídios intercelulares é a responsável direta por evitar a perda de água transcutânea. ${ }^{62}$ Assim, pela sua estrutura e composição, o estrato córneo é a principal barreira limitante à difusão percutânea de fármacos.

Um fármaco pode atravessar o estrato córneo através de três diferentes vias (Figura 2). São elas: ${ }^{63,64}$ via intercelular: o fármaco difunde-se ao redor dos corneócitos, permanecendo constantemente dentro da matriz lipídica; via transcelular: o fármaco passa diretamente através dos corneócitos e da matriz lipídica intercelular intermediária; via apêndices: rota paralela, na qual os fármacos podem ser absorvidos pelo folículo piloso, glândulas sebáceas e glândulas sudoríparas.

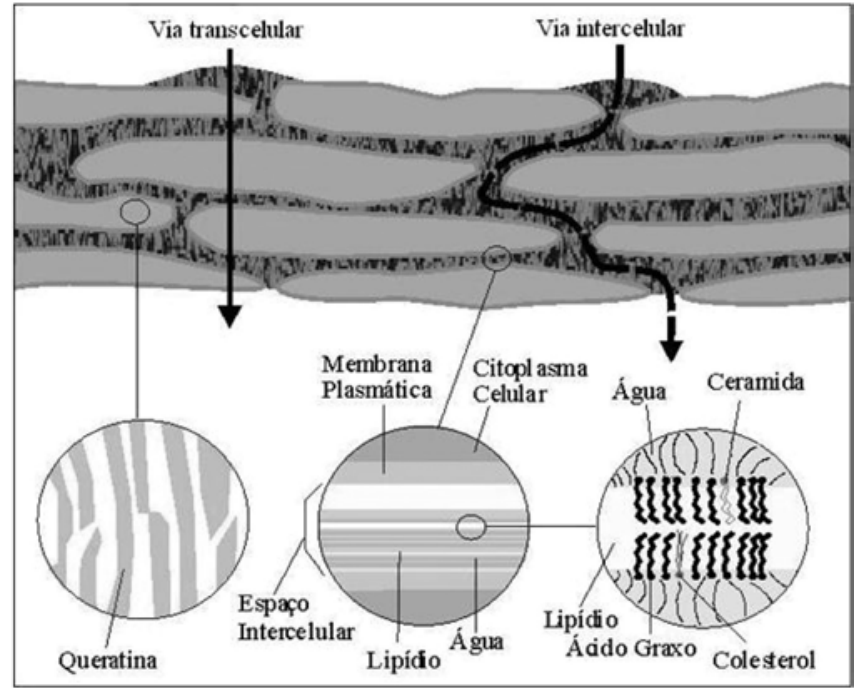

Figura 2. Vias de permeação de fármacos através do estrato córneo: através da matriz lipídica, entre os corneócitos (penetração intercelular) e através dos corneócitos e a matriz lipídica (penetração transcelular) Adaptado da ref. 63

A importância de cada via de permeação depende das propriedades físico-químicas do fármaco e das características da membrana. Em condições normais, ou seja, na ausência de corrente elétrica, a penetração transdérmica de fármacos se restringe a poucas moléculas, que devem ser, necessariamente, potentes (capazes de atingir o efeito terapêutico em baixas concentrações), pequenas (Massa Molar $<400$ $\mathrm{g} / \mathrm{mol}$ ) e ligeiramente lipofílicas (para se partilharem através das camadas da epiderme). Na iontoforese, no entanto, o transporte de substâncias através da pele sob condições moderadas $\left(0,1 \mathrm{~mA} / \mathrm{cm}^{2}\right)$ ocorre principalmente via anexos cutâneos, como folículos pilosos e glândulas sudoríparas. Esses anexos atuam como vias alternativas de baixa resistência ao transporte de substâncias exógenas. ${ }^{65-68}$ Assim, na iontoforese, o estrato córneo deixa de ser a principal barreira relevante 
à penetração, uma vez que as moléculas atravessam a epiderme por "vias alternativas", sendo então possível a liberação de macromoléculas e moléculas polares, ampliando as possibilidades terapêuticas utilizando a via transdérmica.

Ademais, o transporte de íons pela via intercelular também ocorre na iontoforese. Estudos utilizando espectroscopia eletrônica, microscopia de fluorescência e microscopia confocal a laser confirmam a diminuição da resistência do estrato córneo quando este é submetido a uma corrente elétrica fraca. Essa queda na resistência da pele ocorre devido à formação de poros aquosos, ou seja, regiões polares na lamela lipídica. ${ }^{69}$ Fatouros et al. ${ }^{70}$ observaram através de microscopia eletrônica a formação desses poros aquosos em estudos in vitro de iontoforese utilizando pele humana proveniente de cirurgias plásticas. Ainda, em estudos de iontoforese in vivo, esses autores observaram um enfraquecimento nas estruturas dos desmossomos. Os desmossomos são estruturas protéicas que formam uma ponte entre corneócitos vizinhos. Seu enfraquecimento facilita a penetração de substâncias pela pele. As causas deste enfraquecimento ainda não são conclusivas, podendo ter uma relação direta ou indireta com a corrente elétrica. O efeito direto está obviamente relacionado à aplicação da própria eletricidade, que pode fragilizar os desmossomos. O efeito indireto deve-se ao aumento do conteúdo aquoso causado pela aplicação da corrente elétrica.

\section{MECANISMOS DE TRANSPORTE IONTOFORÉTICO}

Existem várias análises detalhadas sobre os mecanismos envolvendo o fenômeno iontoforético e o transporte das moléculas de fármacos através da pele, sendo os dois mais aceitos a eletrorrepulsão e a eletrosmose, ${ }^{52,71-73}$ conforme esquematizado na Figura 3.

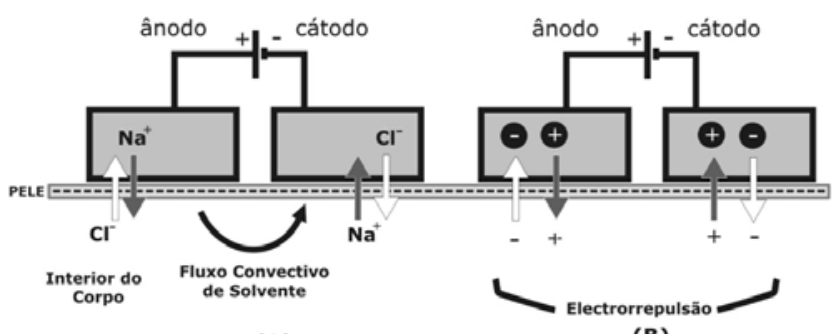

(A)

(B)

Figura 3. Esquema do fluxo eletrosmótico (A) e do fluxo eletrorrepulsivo (B)

A eletrorrepulsão refere-se ao movimento ordenado de íons na presença de uma corrente elétrica aplicada ao meio. O fluxo eletrorrepulsivo de um íon $(x)$ está diretamente relacionado com o fluxo da corrente elétrica $\left(i_{\mathrm{x}}\right)$ (Equação 2$)$.

$$
J_{x}^{E R}=\frac{1}{z_{x} A F} i_{x}
$$

onde: $J_{\mathrm{x}}^{\mathrm{ER}}$ representa o fluxo eletrorrepulsivo do íon $x ; A$ é a área onde ocorrerá o transporte através da pele, $z_{x}$ é a carga do íon $x$ e $F$ é a constante de Faraday $(96.500 \mathrm{C} / \mathrm{mol})$.

O fluxo iônico devido ao movimento do íon $x$ pode ser relacionado à corrente aplicada ( $I$ em Amperes) por uma constante de proporcionalidade $\left(t_{x}\right)$, ou seja, pelo número de transporte de $x$ ( $0<$ $\left.t_{x}<1\right)$ (Equação 3).

$$
J_{x}^{E R}=\frac{1}{z_{x} A F} t_{x} I
$$

O número de transporte é definido como sendo a fração da corrente total que é transportada por um íon específico. ${ }^{74} \mathrm{~A}$ soma dos números de transporte de todos os íons presentes no sistema é igual a $1 .^{48} \mathrm{O}$ número de transporte do íon $x\left(t_{x}\right)$ depende das propriedades físico-químicas deste íon e de como estas se comparam com as propriedades físico-químicas de outras moléculas carregadas presentes no sistema (Equação 4):

$J_{x}^{E R}=\left(\frac{1}{z x F}\right) \frac{z_{x} u_{x} c_{x}}{\sum_{n=0}^{i} z_{i} u_{i} c_{i}} I_{D}$

onde, $I_{D}$ é a densidade de corrente aplicada (I/A) e $z_{x}, u_{x}$ e $c_{x}$ referem-se à carga, mobilidade e concentração do fármaco, respectivamente. No denominador encontra-se a somatória desses parâmetros para cada íon do sistema que contribui para a transferência de cargas através da membrana. ${ }^{56}$ Portanto, considerando-se que os íons presentes no interior da pele também contribuem para a condução da corrente elétrica, o número de transporte de um fármaco jamais será igual a $1 .{ }^{49}$

Através dessas equações é possível deduzir que o transporte da corrente elétrica é competitivo entre os íons presentes no sistema. Portanto, a presença de outros íons pode reduzir o fluxo do fármaco e, conseqüentemente, sua permeação cutânea. ${ }^{75-79}$

O fluxo eletrorrepulsivo de cada íon está relacionado com sua mobilidade elétrica e concentração, como mostra a Equação 4. Dessa forma, aumentando-se a concentração de moléculas competidoras de alta mobilidade no sistema, o fluxo do fármaco é certamente diminuído, e o inverso ocorre quando se compara o fluxo deste fármaco na presença de moléculas competidoras com baixa mobilidade e mesma concentração. ${ }^{80-82}$

Por exemplo, Sebastiani et al. ${ }^{83}$ avaliaram a influência da presença do ácido lático na iontoforese do ibuprofeno. A iontoforese foi capaz de aumentar a liberação do ibuprofeno; no entanto, quando o ácido lático foi adicionado ao cátodo houve diminuição do fluxo do fármaco, provavelmente pela competição dessas moléculas pelo transporte de corrente elétrica. ${ }^{81,84}$ Abla et al. ${ }^{52}$ avaliaram o efeito de íons $\mathrm{Na}^{+}$na liberação iontoforética in vitro de um dipeptídeo modelo (D-(Arg)-kyotorphine). Os experimentos com níveis reduzidos de $\mathrm{Na}^{+}$foram conduzidos utilizando-se uma ponte salina separando o compartimento contendo o eletrodo e o $\mathrm{NaCl}$ (necessário para as reações eletroquímicas dos eletrodos) do compartimento contendo a formulação e o peptídeo, mas, ao mesmo tempo, permitindo o fluxo elétrico entre os dois compartimentos. Os autores observaram que o fluxo do peptídeo, quando a ponte salina foi empregada, foi cerca de 5 vezes maior que aquele realizado sem ponte salina devido à diminuição da competição direta do fármaco com o $\mathrm{Na}^{+}$. Trabalho semelhante foi conduzido por Cázares-Delgadillo et al.. ${ }^{71} \mathrm{Na}$ ausência de ponte salina, contendo aproximadamente $170 \mathrm{mM}$ de $\mathrm{NaCl}$ na formulação doadora, observaram um decaimento significativo no fluxo iontoforético do Citocromo c, uma proteína pequena $(12,4$ $\mathrm{KDa}$ ), em relação aos experimentos realizados com a ponte salina separando o $\mathrm{NaCl}$ do contato com a proteína. Os autores salientaram ainda que o fluxo eletrosmótico permaneceu constante, sendo este decaimento no fluxo eletrorrepulsivo causado pela presença de íons competidores.

A situação ideal para a iontoforese seria a de single-ion, ou seja, a presença somente do fármaco na formulação. Nesta circunstância a molécula de interesse competiria apenas com os contra-íons endógenos. No entanto na prática, esta situação encontra limitações, uma vez que formulações farmacêuticas incorporam, na maioria das vezes, aditivos carregados, como tampões, modificadores de viscosidade e conservantes, que podem prejudicar a eficiência do transporte da molécula de interesse. ${ }^{50}$ 
Além da eletrorrepulsão, outro mecanismo de transporte iontoforético bastante estudado é a eletrosmose. A eletrosmose refere-se ao fluxo de um volume de solvente e movimentação de cargas quando uma diferença de potencial elétrico é aplicada na pele. O termo eletrosmótico deriva do fato de que, sobre a influência da corrente elétrica, a pele favorece o transporte do $\mathrm{Na}^{+}$sobre o $\mathrm{Cl}^{-}$devido à permesseletividade desta membrana. Isto é, a pele possui ponto isoelétrico de aproximadamente 4,0-4,5 e, acima desta faixa de $\mathrm{pH}$, os grupos carboxilatos associados a resíduos de aminoácidos presentes na membrana encontram-se ionizados. Portanto, a pele se encontra carregada negativamente quando em contato com uma solução com $\mathrm{pH}$ fisiológico ${ }^{85,86}$ favorecendo o transporte de cátions e dificultando o de ânions. Vários autores demonstraram que o número de transporte do $\mathrm{Na}^{+}$através da pele humana in vitro, em $\mathrm{pH} 7,4$, é quase o dobro do número de transporte do $\mathrm{Cl}^{-}{ }^{87,88}$

Sendo assim, a aplicação de um campo elétrico através da pele, favorece o movimento de íons no sentido do ânodo para o cátodo, na tentativa de neutralizar as cargas negativas desta membrana. ${ }^{89}$ Estes íons, ao se movimentarem, levam consigo um certo volume de solvente e este impulso é transferido para as moléculas neutras presentes no sistema. Portanto, o fluxo de solvente ou fluxo eletrosmótico faz com que moléculas neutras possam ser liberadas por iontoforese através do ânodo ${ }^{23}$ e os cátions se beneficiem desta segunda força adicional à eletrorrepulsão. ${ }^{55,71}$

De maneira geral, a mobilidade elétrica de uma molécula é inversamente proporcional ao seu tamanho e, pelo fato de moléculas grandes também serem levadas pelo fluxo de solvente, aproveitando-se das vias de baixa resistência do tecido, a eletrosmose desempenha papel fundamental na permeação cutânea de macromoléculas. ${ }^{52,71,90,91}$

Considerando que o fluxo iontoforético total $(J)$ de uma molécula se dá pela adição das contribuições dos fluxos passivo, eletrorrepulsivo e eletrosmótico, Guy et al..$^{82}$ apresentaram graficamente quais seriam as contribuições de cada mecanismo no fluxo iontoforético de fármacos catiônicos em função da massa molar (Figura 4).

Primeiramente deve-se ressaltar que as curvas apresentadas no gráfico da Figura 4 são simulações, com o propósito de melhor visualização, não havendo deduções quantitativas. Por esta razão os fluxos relativos calculados estão apresentados em função da massa

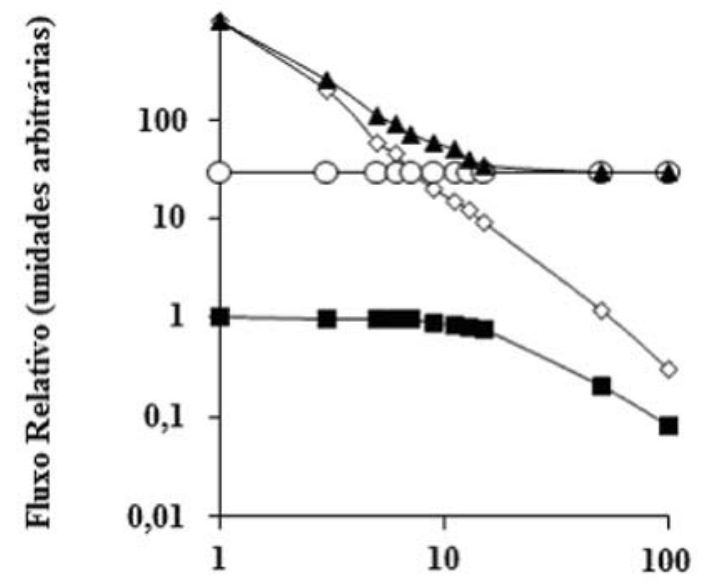

\section{Massa Molecular Relativa}

Figura 4. Contribuição dos fluxos passivo, eletrorrepulsivo e eletrosmótico ao transporte iontoforético total de fármacos catiônicos em função da sua massa molecular relativa. $\mathbf{\square}$ fuxo passivo; $\diamond$ fuxo eletrorrepulsivo; $\bigcirc$ fluxo eletrosmótico; $\Delta$ fluxo total. Adaptado da ref. 82 molar (MM) relativa da molécula. É possível notar nessa Figura a pequena contribuição da permeação passiva, e que esta contribuição diminui proporcionalmente com o aumento da MM. Quando se compara o fluxo passivo com os fluxos eletrorrepulsivo e eletrosmótico de moléculas pequenas observa-se que a eletrorrepulsão provoca um aumento de aproximadamente 100 vezes no fluxo, enquanto que a eletrosmose tem uma contribuição mais modesta, em torno de 30 unidades arbitrárias. No entanto, quando a contribuição de cada fluxo para o transporte de moléculas de grande MM é analisada observa-se que, em relação ao fluxo passivo, a eletrorrepulsão provoca um aumento de apenas 10 vezes no fluxo, contribuição esta bem menor que aquela apresentada para moléculas pequenas. O fluxo eletrosmótico passa então a ser dominante, uma vez que continua constante.

\section{INFLUÊNCIA DA FORMULAÇÃO NA IONTOFORESE}

\section{Forma farmacêutica}

A forma farmacêutica utilizada para a liberação iontoforética de um fármaco pode ser líquida ou semi-sólida, mas deve sempre ser hidrofílica para permitir a passagem da corrente elétrica. Assim, soluções aquosas são normalmente utilizadas para incorporar o fármaco que se deseja liberar. No entanto, géis hidrofílicos também têm a capacidade de conduzir corrente elétrica e, por terem uma certa viscosidade, permanecem no local de aplicação, não escorrendo como ocorre com as soluções. ${ }^{92}$ A garantia de que a formulação permanecerá no local de aplicação oferece ao paciente maior liberdade de movimento durante o tratamento. ${ }^{81}$

Géis de polímero carboxivinílico, os quais possuem características aniônicas, por exemplo, têm sido muito estudados e aprovados para uso farmacêutico em diferentes locais de administração. Sua utilização como veículo para a iontoforese pode ser vantajosa, uma vez que possuem boas propriedades reológicas, permanecendo no local por longos períodos, ${ }^{93}$ são inertes em condições fisiológicas, atóxicos e possuem excelente estabilidade térmica. ${ }^{94}$ Além disso, géis aniônicos normalmente possuem boa capacidade tamponante, contribuindo para a manutenção do $\mathrm{pH}$ desejado (entre 5,0 e 7,0). ${ }^{81}$

Géis hidrofílicos não iônicos também são uma boa alternativa às soluções para a iontoforese. O gel de hidroxietilcelulose (HEC), por exemplo, é utilizado para produzir formulações com uma grande variedade de viscosidades e sua capacidade de dissolução não é afetada pela presença de íons no sistema. ${ }^{95}$

O polímero escolhido para a obtenção do gel deve ser obviamente compatível com o fármaco e não interferir em sua liberação. A liberação rápida do fármaco do gel permite sua maior disponibilidade na superfície da pele, possibilitando que ele se difunda através desta membrana. Sendo assim, deve-se levar em consideração a carga elétrica apresentada pelo fármaco quando incorporado no gel. Isto porque interações do ativo com o polímero podem diminuir sua liberação da formulação. Tatavarti et al. ${ }^{96}$ estudaram a influencia do gel do polímero carboxivinílico na liberação de duas bases fracas, a papaverina e o verapamil, em pH 6,8, quando elas estavam positivamente carregadas, e verificaram que o polímero, por interagir eletrostaticamente com as moléculas desses dois fármacos, sustentava sua liberação. Fato semelhante a este foi observado por Gelfuso et al. ${ }^{97} \mathrm{com}$ a incorporação de uma porfirina carregada positivamente no mesmo polímero aniônico, e o oposto quando uma porfirina negativamente carregada foi incorporada. O gel aniônico parece favorecer a liberação da porfirina negativa possivelmente devido a uma repulsão entre as cargas da molécula e as cargas de igual polaridade do polímero carboxivinílico. Prasad et $a l .{ }^{51}$ estudaram a liberação iontoforética do metotrexato sob diferentes parâmetros elétricos a partir de géis hidrofílicos de acrilamida e ácido acrílico. Observaram que, aumentando-se a quantidade de ligações 
cruzadas nos polímeros, o fluxo do fármaco aumentava. Este fato foi atribuído ao maior acúmulo de fármaco na superfície do gel. Os autores observaram ainda uma diminuição do fluxo de liberação do fármaco da formulação quando o conteúdo de ácido acrílico era aumentado e sugeriram possível interação entre um grupamento amina do metotrexato com a função ácido do copolímero.

A viscosidade dos géis também deve ser avaliada, uma vez que a migração de íons está inversamente relacionada com a viscosidade do veículo em que eles estão contidos..$^{51,98}$ Gratieri et al. ${ }^{99}$ avaliaram a iontoforese do DMAE $p$-acetamidobenzoato, utilizado como tensor facial em tratamentos antienvelhecimento, incorporado em gel semisólido não iônico de HEC. Verificaram que a incorporação do fármaco no gel diminuiu aproximadamente 2,5 vezes seu fluxo iontoforético quando comparado com uma solução aquosa.

\section{Concentração do fármaco}

A concentração do fármaco na formulação e a sua correlação com o fluxo iontoforético é um dos parâmetros mais estudados atualmente..$^{40,71,76,81,100}$ Embora as Equações 2 a 4 demonstrem esta correlação, os resultados experimentais não a confirmam em determinados casos, evidenciando sua complexidade. Sabe-se que ao se aumentar a concentração do fármaco na formulação não se aumenta necessariamente sua quantidade na pele e, neste caso, deve-se considerar a influência dos íons competidores, as características físicoquímicas da molécula do fármaco e sua capacidade de interagir com outras estruturas ao longo dos estratos cutâneos..$^{56}$

Ao se considerar fármacos para os quais a eletrorrepulsão é dominante, seu número de transporte, ou seja, seu fluxo, tende a aumentar com o aumento de sua concentração quando outros íons além do fármaco estão presentes na formulação. No entanto, quando a iontoforese do fármaco é realizada na ausência de co-íons, o íon de interesse compete apenas com contra-íons endógenos. Neste caso, o número de transporte do fármaco atinge um valor máximo independente de sua concentração. Patel et al. ${ }^{40}$ avaliaram a liberação iontoforética do sumatriptano succinato a partir de um patch. Este patch foi desenvolvido de forma que os co-íons ficassem em um compartimento separado do fármaco. Sendo assim, o compartimento contendo o eletrodo de $\mathrm{Ag}$ (ânodo) continha uma pequena quantidade de cloreto de sódio e uma resina de troca iônica que prevenia os íons formados de competirem com o fármaco pelo transporte da corrente elétrica. O compartimento contendo o eletrodo era separado do compartimento contendo a formulação do fármaco por uma membrana poro-seletiva (poros de $100 \mathrm{Da}$ ). Os autores observaram que, nesse sistema, um aumento da quantidade de fármaco de 9,7 para $30 \mathrm{mg}$ não resultou em aumento significativo do fluxo. Este estudo confirma a teoria exposta anteriormente: quando a iontoforese do fármaco é feita na ausência de co-íons, o número de transporte do fármaco atinge um valor máximo independente de sua concentração. A eficiência de liberação do fármaco, ou seja, a fração de corrente transportada por seus íons, é então determinada pela sua difusividade na pele em relação à difusividade do contra-íon predominante (normalmente $\mathrm{Cl}^{-}$) do lado oposto da pele. Como se espera que a difusividade do fármaco e do $\mathrm{Cl}^{-}$sejam independentes de suas concentrações, a eficiência de liberação do fármaco conseqüentemente não é afetada por este parâmetro. ${ }^{48,56,73,101}$

Marro et al. ${ }^{73}$ por exemplo, estudaram a liberação iontoforética in vitro da lidocaína, propranolol e quinina, variando suas concentrações, e obtiveram um aumento linear de seus respectivos fluxos através da pele. No entanto, na ausência de íons competitivos, os fluxos iontoforéticos dos três fármacos se mostraram independentes de suas concentrações no compartimento doador. Isso porque as moléculas do fármaco eram as únicas espécies catiônicas no sistema capazes de transportar a corrente elétrica através da pele, ou seja, seu número de transporte já era máximo.

Ao se considerar fármacos para os quais o fluxo eletrosmótico é dominante, a correlação pode ser ainda mais complexa para certas moléculas lipofílicas catiônicas, como a nafarelina e a leuprolida, em que se aumentando a concentração observou-se a redução da quantidade liberada. Isto porque a eletrosmose pode ser significantemente atenuada na liberação destas moléculas, uma vez que elas podem reduzir, neutralizar, ou até mesmo reverter a carga negativa da pele em pH fisiológico, devido à sua grande afinidade pelos sítios carregados da membrana. ${ }^{82}$

\section{Influência do pH da formulação}

$\mathrm{Na}$ iontoforese, fármacos que apresentam carga elétrica no $\mathrm{pH}$ da formulação devem ser, em princípio, colocados no compartimento próximo ao eletrodo de igual polaridade. Fármacos que não apresentam carga elétrica, dispersos em formulações com pH fisiológico, são colocados inicialmente em contato com o eletrodo positivo visando a contribuição do fluxo eletrosmótico em seu transporte transcutâneo.

A variação do $\mathrm{pH}$ de uma formulação doadora de fármaco pode aumentar ou não a contribuição eletrosmótica no fluxo de uma molécula através da pele, além de determinar o grau de ionização da própria molécula de fármaco. Ao se aumentar o $\mathrm{pH}$ de uma formulação contendo uma base fraca, por exemplo, a fração ionizada da base diminui, diminuindo o potencial eletromigratório. No entanto, em valores mais altos de $\mathrm{pH}(6,5-7,0)$, a eletrosmose passa a contribuir de maneira mais significativa, uma vez que a membrana (pele) se encontra totalmente ionizada. ${ }^{80} \mathrm{O}$ impacto dessas forças oponentes dependerá das propriedades físico-químicas de cada molécula a ser estudada.

Abla et al..$^{53}$ investigaram o impacto da carga e do peso molecular na liberação iontoforética de uma série de dipeptídeos. Observaram que, para peptídeos com massa molar semelhante, a maior valência determinava um maior fluxo. Portanto, para peptídeos zwiteriônicos, o fluxo iontoforético é dependente do $\mathrm{pH}$ da formulação.

Kochhar e Imanidis ${ }^{77}$ estudaram a liberação iontoforética da leuprolida, um peptídeo agonista de LHRH. Relataram que quando o $\mathrm{pH}$ da formulação que continha o fármaco foi aumentado de 4,5 para 7,2 , a quantidade de leuprolida permeada foi quase o dobro, mesmo com suas molécula estando bem menos ionizadas neste $\mathrm{pH}$. Pode-se inferir que a eletrosmose, neste caso, foi bastante significante para a liberação do peptídeo.

Merino et al. ${ }^{86}$ delinearam, em seu trabalho, as contribuições da eletrorrepulsão e da eletrosmose ao fluxo iontoforético do 5-Fluorouracil (5-FU) através da pele de suínos in vitro. Em pH 8,5 o fármaco negativamente carregado (pKa 8) apresentou transporte catódico eletrorrepulsivo muito maior que o transporte anódico, que se daria por eletrosmose, já que o fármaco negativo não seria repelido quando em contato com o eletrodo positivo. Ao reduzirem o pH do meio para 6,0 e 5,0, houve redução da porcentagem de fármaco ionizado, o transporte catódico eletrorrepulsivo diminuiu e a eletrosmose, na direção ânodo-cátodo, passou a ser o mecanismo dominante através da pele carregada negativamente. No entanto, em pH 4,0, os fluxos anódicos e catódicos foram equilibrados, sugerindo a neutralidade da pele sob estas condições (i.e., pI 4,0 ). Este fato foi confirmado quando o $\mathrm{pH}$ foi ajustado a 3,0 e a eletrosmose, no sentido cátodo-ânodo, passou a ser o mecanismo dominante através da pele então carregada positivamente. Com este experimento Merino et al..$^{86}$ determinaram que o ponto isoelétrico (pI) da pele de suínos está em torno de 4,0. Em 2001, Marro et al..$^{73}$ confirmaram este $\mathrm{pI}$ medindo o fluxo de um fármaco modelo em condições 
"simétricas" (isto é, em uma situação "artificial" em que o pH das soluções dos dois lados da membrana foi alterado). Observaram que a pele de suínos e de humanos se torna uma membrana "sem" carga (carga líquida $=0$ ) na faixa de $\mathrm{pH}$ de 4,0 a 4,5, o que levou, portanto, à diminuição ou ausência do fluxo eletrosmótico nessa faixa de $\mathrm{pHs}$.

Lopez et al..$^{24}$ avaliaram o transporte iontoforético anódico in vitro do ácido 5-aminolevulínico (ALA) em função do pH da formulação. Dada a natureza principalmente zwiteriônica do ALA em pH 7,4, o mecanismo de eletrotransporte apresentado por esta molécula quando colocada em contato com o eletrodo positivo foi o eletrosmótico, uma vez que o fármaco não tem carga líquida neste $\mathrm{pH}$ para sofrer eletrorrepulsão. No entanto, a diminuição do $\mathrm{pH}$ da formulação para aumentar a fração do ALA na forma catiônica, e assim a eletrorrepulsão, não resultou em aumento do transporte do fármaco. Isto aconteceu porque o aumento do componente eletrorrepulsivo foi cancelado por uma diminuição do fluxo eletrosmótico conforme as cargas negativas da pele foram neutralizadas pelo $\mathrm{pH}$ ácido da formulação. Portanto, o pH ótimo da formulação deve ser avaliado levando-se em consideração o grau de ionização da molécula do fármaco, que comanda o fluxo eletrorrepulsivo, e a ionização da pele, que comanda o fluxo eletrosmótico. A partir das contribuições relativas de cada mecanismo em um determinado $\mathrm{pH}$, pode-se concluir qual compartimento (ânodo ou cátodo) é mais adequado para inserção do fármaco.

\section{DISPOSITIVOS ATUALMENTE UTILIZADOS E POSSIBILIDADES FUTURAS}

$\mathrm{O}$ interesse atual pela iontoforese pode ser demonstrado pelo

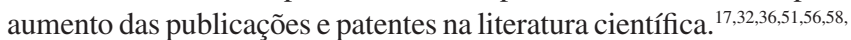
65,91,100,102-107 Entretanto, até 1993, a iontoforese havia sido aprovada pelo conselho norte-americano (FDA) somente como um teste diagnóstico para a fibrose cística ${ }^{108}$ e para o tratamento de hiperidrose. ${ }^{109}$ Em maio de 2004, o FDA anunciou a aprovação do LidoSite ${ }^{\circledR}$ Topical System da Vyteris, um dispositivo de aplicação iontoforética de lidocaína e epinefrina. $O$ produto foi aprovado para uso em pacientes acima de 5 anos de idade. Em junho de 2004, a Alza recebeu aprovação do FDA para o dispositivo iontoforético Ionsys ${ }^{\circledast}$, para liberação de fentanil, um analgésico.

Atualmente os dispositivos iontoforéticos são miniaturizados ${ }^{110}$ (Figura 5) e colocados na pele da mesma maneira que os tradicionais sistemas transdérmicos passivos. São constituídos por três compartimentos: reservatório do fármaco, reservatório de retorno e controlador eletrônico. Quando o dispositivo é aplicado à pele, o controlador eletrônico, que contém uma bateria e um microcomputador programável, inicia a passagem de uma corrente elétrica entre os eletrodos. O fármaco é repelido do reservatório diretamente para a pele e corrente circulatória. O reservatório de retorno contém uma solução salina para fechar o circuito elétrico. ${ }^{41,111}$

Além dos dispositivos já prontos, contendo formulações otimizadas do fármaco, existem no mercado vários dispositivos em que o compartimento contendo o eletrodo é preenchido com uma solução

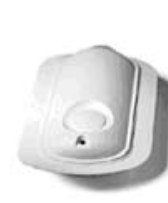

(A)

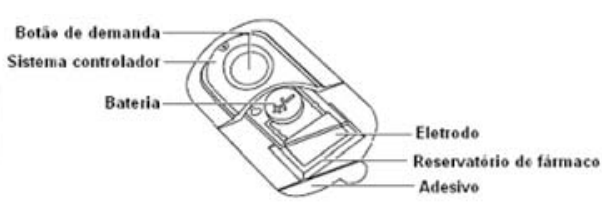

(B)
Figura 5. Dispositivo iontoforético E-Trans ${ }^{\circledR}$ (Alza Company, www.alza. com, 2006) aquosa do fármaco somente antes do uso. A Iomed desenvolveu uma grande quantidade deles baseados no sistema GelSponge ${ }^{\circledR}$, no qual os eletrodos permanecem embebidos em um hidrogel. Quando este compartimento é preenchido pelo fármaco a solução é geleificada e muda sua coloração, indicando que os eletrodos estão devidamente hidratados e, portanto, propícios para a condução da corrente elétrica, e que toda esta superfície entrará em contado com a pele. Os eletrodos são então conectados a uma fonte de energia portátil e a corrente elétrica adequada (máximo de $0,5 \mathrm{~mA} / \mathrm{cm}^{2}$ ) é aplicada. ${ }^{112}$

A iontoforese tem sido clinicamente aplicada no tratamento da hiperidrose, ${ }^{16}$ na administração de anestésicos locais antes de punções na pele ou outros procedimentos cutâneos ${ }^{34,113}$ e no tratamento de disfunções inflamatórias músculo-esqueléticas, tanto com o uso de antiinflamatórios não-esteroidais quanto com corticosteróides. ${ }^{75}$

Apesar do uso clínico aparentemente limitado a poucos fármacos, vários estudos abrangem diversas classes terapêuticas. Podem ser encontrados na literatura estudos envolvendo a penetração transdérmica de peptídeos, como o hormônio luteinizante ${ }^{114,115}$ e a insulina, ${ }^{116-118} \mathrm{de}$ bases, nucleosídeos e nucleotídeos, ${ }^{42,119}$ de anti-hipertensivos, ${ }^{120-122} \mathrm{de}$ glicocorticóides ${ }^{29,123}$ e de antibióticos. ${ }^{124-126}$

A iontoforese também tem sido estudada para melhorar a eficácia tópica, local, de diversas substâncias. Ebihara et al. ${ }^{127}$ estudaram, in vitro, a liberação tópica de ácido ascórbico através da pele de rato utilizando a iontoforese. O ácido ascórbico é utilizado no combate ao envelhecimento cutâneo, por possuir atividade antioxidante, além de atividade protetora contra as radiações UVA e UVB. O estudo concluiu que o tratamento tópico pode ser viabilizado com o uso da iontoforese. Kavanagh e Shamis ${ }^{128}$ demonstraram, in vivo, a possibilidade de se liberar toxina botulínica em quantidades terapêuticas para o tratamento de hiperidrose, utilizando uma unidade pequena de iontoforese (Iomed Phoresor ${ }^{\circledR} I I$ ). Pacini et al. ${ }^{129}$ demonstraram que o ácido hialurônico, utilizado no tratamento de diversas patologias cutâneas, pode atingir grandes concentrações na derme quando liberado por iontoforese, sendo este método uma alternativa segura em substituição às injeções.

A iontoforese também vem sendo estudada associada à Terapia Fotodinâmica (TFD) para aumentar a penetração do ácido 5-aminolevulínico (ALA), precursor da protoporfirina IX, que é um potente fotossensibilizador, ${ }^{22-24,130,131}$ e da meso-tetra(N-metil, 4-piridil)-porfirina (TMPyP), uma porfirina carregada positivamente. ${ }^{97,132}$

\section{CONCLUSÃO}

O desenvolvimento de formulações adequadas deve levar à otimização da eficiência da corrente e, conseqüientemente, ao aumento da quantidade de fármaco liberado na pele ou através dela. Assim, apesar dos desafios atualmente encontrados, a iontoforese é uma técnica cada vez mais promissora. A incorporação de microprocessadores tem sido um importante avanço na garantia de administração segura e eficaz de fármacos potentes. É possível prever sua larga utilização tanto para a liberação transdérmica como tópica de diferentes fármacos.

\section{AGRADECIMENTOS}

À Fundação de Amparo à Pesquisa do Estado de São Paulo (FAPESP) pelo auxílio financeiro.

\section{REFERÊNCIAS}

1. Panchagnula, R.; Pillai, O.; Nair, V. B.; Ramarao, P.; Curr. Opin. Chem. Biol. 2000, 4, 468.

2. Chien, Y.W.; Banga, A.K.; J. Pharm. Sci. 1989, 78, 353.

3. Greenbaum, S. S.; Dermatol. Surg. 2001, 12, 1027. 
4. Giannola, L. I.; De Caro, V.; Giandalia, G.; Siragusa, M. G.; Campisi, G.; Florena, A. M.; Ciach, T.; Eur. J. Pharm. Biopharm. 2007, 65, 238.

5. Jacobsen, J.; J. Controlled Release 2001, 70, 83.

6. Bose, W.; Dtsch. Zahnarztl Z. 1991, 46, 806.

7. Murthy, K. S.; Tailim, S. T.; Singh, I.; Oral Surg. Oral Med. Oral Pathol. 1973, 36, 448.

8. Schaeffer, M. L.; Bixler, D.; Yu, P.; J. Periodontal. 1971, 42, 695.

9. Molokhia, S. A.; Jeong, E. K.; Higuchi, W. I.; Li, S. K.; Int. J. Pharm. 2007, 335, 46.

10. Eljarrat-Binstock, E.; Domb, A. J.; J. Controlled Release 2006, 110, 479.

11. Yasukawa, T.; Ogura, Y.; Tabata, Y.; Kimura, H.; Wiedemann, P.; Honda, Y.; Prog. Retin. Eye Res. 2004, 23, 253.

12. Sarraf, D.; Lee, D. A.; J. Ocul. Pharmacol. 1994, 10, 69.

13. Church, A. L.; Barza, M.; Baum, J.; Invest. Ophthalmol. Vis. Sci. 1992, 33,3543 .

14. Murthy, S. N.; Waddell, D. C.; Shivakumar, H. N.; Balaji, A.; Bowers, C. P.; J. Dermatol. Sci. 2007, 46, 150.

15. Murthy, S. N.; Wiskirchen, D. E.; Bowers, C. P.; J. Pharm. Sci. 2007, 96, 305.

16. Na, G. Y.; Park, B. C.; Lee, W. J.; Park, D. J.; Kim, W.; Kim, M. N.; Dermatol. Surg. 2007, 33, 57.

17. Morrel, E. M.; Spruance, S. L.; Goldberg, D. I.; Clin. Infect. Dis. 2006, 43,460 .

18. Sintov, A. C.; Brandys-Sitton, R.; Int. J. Pharm. 2006, 316, 58.

19. Alvarez-Figueroa, M. J.; Araya-Silva, I.; Diaz-Tobar, C.; Pharm. Dev. Technol. 2006, 11, 371.

20. Alvarez-Figueroa, M. J.; Blanco-Mendez, J; Int. J. Pharm. 2006, 215, 57.

21. Abla, N.; Naik, A.; Guy, R. H.; Kalia, Y. N.; Pharm. Res. 2006, 23, 1842.

22. Lopez, R. F. V.; Bentley, M. V. L. B.; Delgado-Charro, M. B.; Salomon, D.; van Den Bergh, H.; Lange, N.; Guy, R. H.; Photochem. Photobiol. 2003, 77, 304

23. Lopez, R. F. V.; Bentley, M. V. L. B.; Delgado-Charro, M. B.; Guy, R. H.; J. Controlled Release 2003, 88, 65.

24. Lopez, R. F. V.; Bentley, M. V. L. B.; Delgado-Charro, M. B.; Guy, R. H.; Pharm. Res. 2001, 18, 311.

25. Kanebako, M.; Inagi, T.; Takayama, K.; Biol. Pharm. Bull. 2003, 26, 518.

26. Yoshida, N. H.; Roberts, M. S.; Adv. Drug. Del. Rev. 1992, 9, 239.

27. Green, P. G.; Flanagan, M.; Shroot, B.; Guy, R. H. Em Pharmaceutical skin penetration enhancement; Walters, K. A.; Hadgraft, J., eds.; Marcel Dekker Inc.: London, 1993, vol. 59.

28. Kumar, R.; Philip, A.; Trop. J. Pharm. Res. 2007, 6, 633.

29. Sreerekha, R. R.; Shanmuga, S. V.; Karthick, S. P.; Srinivas, C. R.; Mathew, A. C.; Indian J. Dermatol. Venereol. Leprol. 2006, 72, 283.

30. Cross, S. E.; Roberts, M. S.; Curr. Drug. Delivery 2004, 1, 81.

31. Singh, J.; Gross, M.; Sage, B.; Davis, H. T.; Maibach, H. I.; Food Chem. Toxicol. 2001, 39, 1079.

32. Jadoul, A.; Bouwstra, J. A.; Preat, V.; Adv. Drug Deliv. Rev. 1999, 35, 89.

33. Greenbaum, S. S.; Dermatol. Surg. 2001, 12, 1027.

34. Power, I.; Br. J. Anaesth. 2007, 98, 4.

35. Bain, K. T.; J. Opioid Manag. 2006, 2, 314.

36. Dixit, N.; Bali, V.; Baboota, S.; Ahuja, A.; Ali, J.; Curr. Drug Delivery 2007, 4,1

37. Singh, J.; Gross, M.; Sage, B.; Davis, H. T.; Maibach, H. I.; Food Chem. Toxicol. 2000, 38, 717

38. Oh, S. Y.; Jeong, S. Y.; Park, T. G.; Lee, J. H.; J. Controlled Release 1998, 51,161.

39. Degim, I. T.; Celebi, N.; Curr. Pharm. Des. 2007, 13, 99.
40. Patel, S. R.; Zhong, H.; Sharma, A.; Kalia, Y. N.; Eur. J. Pharm. Biopharm. 2007, 66, 296.

41. Subramony, J. A.; Sharma, A.; Phipps, J. B.; Int. J. Pharm. 2006, 317, 1.

42. Geest, R. V. D.; Hueber, F.; Szoka, F. C. Jr.; Guy, R. H.; Pharm. Res. 1996, 13, 553.

43. Nestor, J. J. J.; Ho, T. L.; Simpson, R. A.; Horner, B. L.; Jones, G. H.; Mcrae, G. I.; Vickery, B. H.; J. Med. Chem. 1982, 25, 795.

44. Herndon, C. M.; Pharmacotherapy 2007, 27, 745.

45. Ashburn, M. A.; Stephen, R. L.; Ackerman, E.; Petelenz, T. J.; Hare, B.; Pace, N. L.; Hofman, A.; J. Pain Symptom Manage. 1992, 7, 27.

46. Hartrick, C. T.; Bourge, M. H.; Gargiulo, K.; Damaraju, C. V.; Vallow, S.; Hewitt, D. J.; Reg. Anesth. Pain Med. 2006, 31, 546.

47. Viscusi, E. R.; Reynolds, L.; Chung, F.; Atkinson, L. E.; Khanna, S.; JAMMA 2004, 291, 1333.

48. Mudry, B.; Guy, R. H.; Delgado-Charro, M. B.; J. Pharm. Sci. 2006, 95 , 561.

49. Mudry, B.; Guy, R. H.; Delgado-Charro, M. B.; Biophys. J. 2006, 90, 2822.

50. Mudry, B.; Guy, R. H.; Delgado-Charro, M. B.; J. Controlled Release 2006, 111, 362 .

51. Prasad, R.; Koul, V.; Anand, S.; Khar, R. K.; Int. J. Pharm. 2007, 333, 70.

52. Abla, N.; Naik, A.; Guy, R. H.; Kalia, Y. N.; J. Controlled Release 2005, 108, 319.

53. Abla, N.; Naik, A.; Guy, R. H.; Kalia, Y. N.; Pharm. Res. 2005, 22, 2069.

54. Fang, J. Y.; Sung, K. C.; Hu, O. Y.; Chen, H. Y.; Arzneim. Forsch. 2001, 51, 408.

55. Cullander, C.; Rao, G.; Guy, R. H.; Prediction of Percutaneous Penetration 1993, 33, 381.

56. Kalia, Y. N.; Naik, A.; Garrison, J.; Guy, R. H.; Adv. Drug. Deliv. Rev. 2004, 56, 619 .

57. Chang, S. L.; Hofmann, G. A.; Zhang, L.; Deftos, L. J.; Banga, A. K.; Int. J. Pharm. 2000, 200, 107.

58. Tiwari, S. B.; Udupa, N.; Int. J. Pharm. 2003, 260, 93.

59. Chien,Y. W.; Novel drug delivery systems, $2^{\text {nd }}$ ed., M. Dekker: New York, 1992.

60. Junqueira, L. C. ; Carneiro, J.; Histologia Básica, 9a ed., Guanabara Koogan: Rio de Janeiro, 2004.

61. Pirot, F.; Berardesca, E.; Kalia, Y. N.; Singh, M.; Maibach, H. I.; Guy, R. H.; Pharm. Res. 1998, 15, 492.

62. Fartasch, M.; Microsc. Res. Tech. 1997, 38, 361.

63. Moser, K.; Kriwet, K.; Naik, A.; Kalia, Y. N.; Guy, R. H.; Eur. J. Pharm. Biopharm. 2001, 52, 103.

64. Kitson, N.; Thewalt, J. L.; Acta Derm. Venereol. Suppl. 2000, 208, 12.

65. Schuetz, Y. B.; Carrupt, P. A.; Naik, A.; Guy, R. H.; Kalia, Y.N.; Eur. J. Pharm. Sci. 2006, 29, 53.

66. Lee, R. D.; White, H. S.; Scott, E. R.; J. Pharm. Sci. 1996, 85, 1186.

67. Scott, E. R.; Laplaza, A. I.; White, H. S.; Phipps, J. B.; Pharm. Res. 1993, 10, 1699.

68. Cullander, C.; Guy, R. H.; J. Invest. Dermatol. 1991, 97, 55.

69. Barry, B. W.; Adv. Drug Delivery Rev. 2002, 54, 31.

70. Fatouros, D. G.; Groenink, H. W. M.; Graaff, A. M.; Van Aelst, A. C.; Koerten, H. K.; Bouwstra, J. A.; Eur. J. Pharm. Sci. 2006, 29, 160.

71. Cázares-Delgadillo, J.; Naik, A.; Ganem-Rondero, A.; QuintanarGuerrero, D.; Kalia, Y. N.; Pharm. Res. 2007, 24, 1360.

72. Sieg, A.; Guy, R. H.; Delgado-Charro, M. B.; Biophys. J. 2004, 87, 3344.

73. Marro, D.; Kalia, Y. N.; Delgado-Charro, M. B.; Guy, R. H.; Pharm. Res. 2001, 18, 1701.

74. Wascotte, V.; Delgado-Charro, M. B.; Rozet, E.; Wallemacq, P.; Hubert, P.; Guy, R. H.; Préat, V.; Pharm. Res. 2007, 24, 1131.

75. Fitzpatrick, D.; Corish, J.; Int. J. Pharm. 2006, 325, 90. 
76. Nugroho, A. K.; Li, L.; Dijkstra, D.; Wikström, H.; Danhof, M.; Bouwstra, J. A.; J. Controlled Release 2005, 103, 393.

77. Kochhar, C.; Imanidis, G.; J. Controlled Release 2004, 98, 25.

78. Aramaki, Y.; Arima, H.; Takahashi, M.; Miyazaki, E.; Sakamoto, T.; Tsuchiya, S.; Biol. Pharm. Bull. 2003, 26, 1461.

79. Fang, J. Y.; Sung, K. C.; Lin, H. F.; Fang, C. L.; Int. J. Pharm. 1999, $178,83$.

80. Nair, V.; Panchagnula, R.; Pharm. Res. 2003, 48, 175.

81. Alvarez-Figueroa, M. J.; Delgado-Charro, M. B.; Blanco-Mendez, J.; Int. J. Pharm. 2001, 212, 101.

82. Guy, R. H.; Kalia, Y. N.; Delgado-Charro, M. B.; Merino,V.; Lopez, A.; Marro, D.; J. Controlled Release 2000, 64, 129.

83. Sebastiani, P.; Nicoli, S.; Santi, P.; Int. J. Pharm. 2005, 292, 119.

84. Nugroho, A. K.; Li, G. L.; Danhof, M.; Bouwstra, J. A.; Pharm. Res. 2004, 21, 844.

85. Merino, V.; Kalia, Y. N.; Guy, R. H.; Trends Biotechnol. 1997, 15, 288.

86. Merino, V.; Lopez, A.; Kalia, Y. N.; Guy, R. H.; Pharm. Res. 1999, 16, 758.

87. Lai, P. M.; Roberts, M. S.; J. Controlled Release 1999, 58, 323.

88. Burnette, R. R.; Ongpipattanakul, B.; J. Pharm. Sci. 1987, 76, 765.

89. Bath, B. D.; Scott, E. R.; Phipps, J. B.; White, H. S.; J. Pharm. Sci. 2000, 89, 1537.

90. Boinpally, R. R.; Zhou, S.; Devraj, G.; Anne, P. K.; Poondru, S.; Jasti, B. R; Int. J. Pharm. 2004, 274, 185.

91. Pikal, M. J.; Adv. Drug Deliv. Rev. 2001, 46, 281.

92. Huang, J. F.; Sung, K. C.; Hu, O. Y.; Wang, J. J.; Lin, Y. H.; Fang, J. Y.; Int. J. Pharm. 2005, 297, 162.

93. Merclin, N.; Bramer, T.; Edsman, K.; J. Controlled Release 2004, 98, 57.

94. Rabiskova, M.; Sedlakova, M.; Vitkova, M.; Kuna M.; Ceska Slov. Farm. 2004, 53, 300.

95. Guo, J. H.; Skinner, G. W.; Harcum, W. W.; Barnum, P. E.; Pharm. Sci. Technol. Today 1998, 1, 254.

96. Tatavarti, A. S.; Metha, K. A.; Augsburger, L. L.; Hoag, S. W.; J. Pharm. Sci. 2004, 93, 2319.

97. Gelfuso, G. M.; Lopez, R. F. V.; Controlled Release Society 32nd Annual Meeting Transactions, Miami, Estados Unidos, 2005.

98. Rai, R.; Srinivas, C. R.; Indian J. Dermatol. Venereol. Leprol. 2005, 71, 236.

99. Gratieri, T.; Lopez, R. F. V.; Braz. J. Pharm. Sci. 2005, 41, 466.

100. Chang, S. L.; Banga, A. K.; J. Pharm. Pharmacol. 1998, 50, 635.

101. Kasting, G. B.; Keister, J. C.; J. Controlled Release 1989, 8, 195.

102. Li, S. K.; Zhu, H.; Higuchi, W. I.; Pharm. Res. 2006, 23,1857.

103. Lambrecht, R.; Clarys, P.; Clijsen, R.; Barel, A. O.; Skin Res. Technol. 2006, 3, 211 .
104. Medi, B. M.; Singh, J.; Int. J. Pharm. 2003, 263, 25.

105. Higuchi, W. I.; Li, S. K.; Gahnem, A. H.; Zhu, H.; Song, Y.; J. Controlled Release 1999, 62, 13.

106. Hirvonen, J.; Guy, R. H.; J. Controlled Release 1998, 50, 283.

107. Prausnitz, M. R.; Adv. Drug Deliv. Rev. 1996, 18, 395.

108. Gibson, L. E.; Cooke, R. E.; Pediatrics 1959, 23, 545.

109. Levit, F.; Cutis 1980, 26, 192.

110. http://www.alza.com/alza/etrans, acessada em Janeiro 2006.

111. Green, P. G.; J. Controlled Release 1996, 41, 33.

112. http://www.iomed.com, acessada em Abril 2005.

113. Batheja, P.; Thakur, R.; Michniak, B.; Expert Opin. Drug Deliv. 2006, 3,127 .

114. Raiman, J.; Koljonen, M.; Huikko, K.; Kostiainen, R.; Hirvonen, J.; Eur. J. Pharm. Sci. 2004, 21, 371.

115. Li-Lan, H.; Chen, Y.; Chien, W.; J. Controlled Release 1996, 40, 187.

116. Lassmann-Vague, V.; Raccah, D.; Diabetes Metab. 2006, 32, 513.

117. Tokumoto, S.; Higo, N.; Sugibayashi, K.; Int. J. Pharm. 2006, 325, 13.

118. Kanikkannan, N.; Singh, J.; Ramarao, P.; J. Controlled Release 1999, $59,99$.

119. Bejjani, R. A.; Andrieu, C.; Bloquel, C.; Berdugo, M.; Benezra, D.; Behar-Cohen, F.; Surv. Ophthalmol. 2007, 52, 196.

120. Nair, V.; Panchagnula, R.; Farmaco 2004, 59, 575.

121. Chesnoy, S.; Durand, D.; Doucet, J.; Courraze, G.; J. Controlled Release 1999, 58, 163.

122. Ganga, S.; Ramarao, P.; Singh, J.; J. Controlled Release 1996, 42, 57.

123. Schaffnit, R.; Wicklandt, P.; Moll, F..; J. Controlled Release 1998, 54, 9.

124. Itália, J. L.; Bhardwaj, V.; Kumar, M. N. V. R.; Drug Discovery Today 2006, 11,846 .

125. Khoo, P. P.; Michalak, K. A.; Yates, P. J.; Megson, S. M.; Day, R. E.; Wood, D. J.; J. Bone Joint Surg. Br. 2006, 88, 1149.

126. Frucht-Pery, J.; Mechoulam, H.; Siganos, C. S.; Hadani, P. E.; Shapiro, M.; Domb, A.; Exp. Eye Res. 2004, 78, 745.

127. Ebihara, M.; Akiyama, M.; Ohnishi, Y.; Tajima, S.; Komata, K.; Mitsui, Y.; J. Dermatol. Sci. 2003, 32, 217.

128. Kavanagh, G. M.; Shams, K.; Br. J. Dermatol. 2004, 151, 1093.

129. Pacini, S.; Punzi, T.; Gulisano, M.; Ruggiero, M.; J. Dermatol. Sci. 2006, 44, 169.

130. Bodde, H. E.; Roemele, P. E.; Star, W. M.; Photochem. Photobiol. 2002 , $75,418$.

131. Gerscher, S.; Connelly, J. P.; Beijersbergen Van Henegouwen, G. M.; MacRobert, A. J.; Watt, P.; Rhodes, L. E.; Br. J. Dermatol. 2001, 144, 983.

132. Gelfuso, G. M.; Lopez, R. F. V.; Braz. J. Pharm. Sci. 2005, 41, 428. 